\title{
Multi-wavelength observations revealing the most obscured high energy sources of our Galaxy
}

\author{
Sylvain Chaty ${ }^{* \dagger}$ \\ Laboratoire AIM, CEA/DSM - CNRS - Université Paris Diderot, Irfu/Service d'Astrophysique, \\ Centre de Saclay, Bât. 709, F-91191 Gif-sur-Yvette Cedex, France \\ E-mail: chaty@cea.fr
}

\begin{abstract}
A new type of high-energy binary system has been revealed by the INTEGRAL satellite. These sources are being unveiled by means of multi-wavelength optical, near- and mid-infrared observations. Among these sources, two distinct classes are appearing: the first one is constituted of intrinsically obscured high-energy sources, of which IGR J16318-4848 seems to be the most extreme example. The second one is populated by the so-called supergiant fast X-ray transients, with IGR J17544-2619 being the archetype. We first give here a general introduction on INTEGRAL sources, before reporting on multi-wavelength optical to mid-infrared observations of a sample constituted of 21 INTEGRAL sources. We show that in the case of the obscured sources our observations suggest the presence of absorbing material (dust and/or cold gas) enshrouding the whole binary system. We finally discuss the nature of these two different types of sources, in the context of high energy binary systems, and give a scenario of unification of all these different types of high energy sources, based on their high energy properties.
\end{abstract}

7th INTEGRAL Workshop

September 8-11 2008

Copenhagen, Denmark

\footnotetext{
${ }^{*}$ Speaker.

${ }^{\dagger}$ I would like to thank here my close collaborators F. Rahoui, J. Rodriguez, J. Tomsick, J. A. Zurita Heras, and also P. Filliatre, P.-O. Lagage, I. Negueruela, L. Pellizza and R. Walter for many fruitful work and discussions on the subject of INTEGRAL sources.
} 


\section{Prelude}

Last December it was the $45^{\text {th }}$ anniversary of the discovery of the first X-ray extra-solar source -Sco X-1-, reported on December $1^{\text {st }} 1962$ by Giacconi et al. $(1962)^{1}$. 40 years after these early $\mathrm{X}$-ray ages, the X-ray sky has been extensively observed, but as we will see unexpected discoveries are still to expect! Sco X-1, the first X-ray source in the constellation of Scorpius, became the prototype of Galactic high energy binary systems, in which it is now commonly accepted that a compact objet (neutron star -NS- or black hole -BH-) accretes from a so-called "companion", "primary" or "secondary" star.

We will begin by a short introduction on the distinction between low mass and high mass Xray binaries (LMXBs and HMXBs respectively). We will continue with a review on INTEGRAL sources (called IGRs in the following): the general results obtained by INTEGRAL, the spatial distribution, the modulation and absorption of sources discovered by this satellite. We will then review some of the stellar winds properties, in order to understand the basic theory on SFXTs, which will lead us to what we call here the grand unification of $\operatorname{sgXBs}$.

The following section will describe an extensive multi-wavelength study based on a sample of IGRs, including the classical SFXT IGR J17544-2619, the intermediate SFXT IGR J18483-0311, and the obscured source IGR J16318-4848. We will finish with AX J1749.1-2733, which might be the most obscured BeXRB, before concluding and giving some perspectives.

\subsection{Galactic X-ray binaries}

There are now 300 X-ray binaries known in our Galaxy (Liu et al. 2006; Liu et al. 2007), that we can classify as LMXBs and HMXBs according to the nature of the companion star hosted in the high enery binary system.

\subsubsection{LMXBs}

There are 186 LMXBs (62\% of the total number of high energy binary systems): the companion has a spectral type later than $\mathrm{B}\left(M<1 M_{\odot}\right)$. The mass transfer is done through Roche Lobe filling, via angular momentum loss through the accretion disc. The LMXBs are concentrated in the Galactic bulge due to the fact that the companion stars, being less massive than the Sun, are old stars. The compact object can be either a BH or a NS. When the NS is magnetized, they are Z or Atoll sources according to their hardness-intensity diagrammes.

\subsubsection{HMXBs}

There are 114 HMXBs (38\% of the total number of high energy binary systems): the companion has an $\mathrm{OB}$ spectral type $\left(M>10 M_{\odot}\right)$. The mass transfer occurs either via a decretion disk (Oe/Be systems), or a strong wind or even via Roche lobe filling (in the case of the supergiant X-ray binaries). These systems are concentrated in the Galactic plane. They are separated in 3 distinct groups according to the nature of their companion star:

- The group of Be/X-ray Binaries (BeXBs, also called Be/X-ray transients) constitute the majority of HMXBs. The companion/donor star is in this case a main sequence Be spectral

\footnotetext{
${ }^{1}$ R. Giacconi won the 2002 Physics Nobel prize for this discovery.
} 
type star. The compact object is a NS located on a wide and moderately eccentric orbit, and it is spending little time in close proximity to the dense circumstellar decretion disk surrounding the Be companion (Coe 2000; Negueruela 2004). Transient X-ray outbursts occur when the compact object passes through the Be-star disc, accreting from the low-velocity and high-density stellar wind. It then exhibits hard X-ray spectra during the outburst.

- The group of supergiant X-ray Binaries ( $\mathrm{sgXBs)} \mathrm{is} \mathrm{made} \mathrm{of} \mathrm{binaries} \mathrm{hosting} \mathrm{a} \mathrm{donor} \mathrm{su-}$ pergiant early-type OB star. The compact object is a NS orbiting deep inside the highly supersonic stellar wind (Kaper et al., 2004). The sgXBs are still separated in two distinct groups, according to the accretion process:

- In the wind-fed systems, the X-ray luminosity is powered by accretion from the strong steady radiative stellar wind, creating a persistent X-ray source $\left(L_{x} \sim 10^{35-36} \mathrm{erg} \mathrm{s}^{-1}\right)$. These systems exhibit large variations on short timescales (due to wind inhomogeneities), and a stable flux on the long run. The compact object orbits on a close orbit $\left(P_{\text {orb }}<15 d\right)$ with low eccentricity.

- In the Roche-lobe overflow systems (so-called classical "bright" sources), the matter flows via the inner Lagrangian point forming an accretion disc, giving a high X-ray luminosity $\left(L_{x} \sim 10^{38} \mathrm{erg} \mathrm{s}^{-1}\right.$ ) during outbursts. We point out that Cyg X-1 is the only $\operatorname{sgXB}$ hosting a confirmed $\mathrm{BH}$.

- Finally, the last group of HMXBs is constituted of all the other main sequence or giant type companion HMXBs (including the symbiotic systems).

\subsection{The Corbet Diagramme}

Corbet (1986) reported a plot of the NS spin period versus the orbital period of HMXBs. This plot, now known as the "Corbet Diagramme", shows that the 3 types of HMXB pulsators (BeXBs, wind-fed and Roche-lobe filling sgXBs) segregate into different regions of this diagramme, owing to the complex feedback processes between modulation periods and dominant accretion mechanism.

A correlation is observed in Be systems, due to the fact that they accrete significant angular momentum to be able to form an accretion disc. On the other hand, no correlation is observed in supergiant systems, because of the low net angular momentum of accreted matter: if an accretion disc is formed during accretion of matter, it must be of transitory nature.

\section{And then INTEGRAL arrived...}

The INTEGRAL observatory is an ESA satellite launched on 17 October 2002 by a PROTON rocket on an excentric orbit. It is hosting 4 instruments: $2 \gamma$-ray coded-mask telescopes -the imager IBIS and the spectro-imager SPI, observing in the range $10 \mathrm{keV}-10 \mathrm{MeV}$, with a resolution of 12 and a field-of-view of $19^{\circ}$ - a coded-mask telescope JEM-X (3-100 keV), and an optical telescope (OMC). 
One of the important results of INTEGRAL has been obtained during the deep observation of our Galaxy the Milky Way, allowing to show that the diffuse X-ray background, below $80 \mathrm{keV}$, could be entirely resolved into X-ray point sources (Lebrun et al., 2004).

\subsection{The $\gamma$-ray sky seen by INTEGRAL}

The $\gamma$-ray sky seen by INTEGRAL is very rich, since 499 sources have been detected by $I N$ TEGRAL, reported in the $3^{\text {rd }}$ IBIS/ISGRI soft $\gamma$-ray catalogue, spanning 3.5 years of observations in the 20-100 keV domain (Bird et al., 2007). Among them, 214 were discovered by INTEGRAL, while the remaining 285 were previously known.

Among these sources, there are 147 XRBs (29\% of the total number of INTEGRAL sources), 163 AGNs (33\%), 27 CVs (5\%), and 20 sources of other type (4\%): 12 SNRs, 2 globular clusters, 2 SGRs and 1 GRB. 129 objects still remain unidentified (26\%). The XRBs are separated in 82 LMXBs (16\%) and 78 HMXBs (16\%). Among the HMXBs, there are 24 BeXBs (31\% of the total of HMXBs) and $19 \mathrm{sgXBs}$ (24\% of the total of HMXBs).

It is interesting to follow the evolution of the ratio between BeXBs and $\operatorname{sgXBs}$. During the pre-INTEGRAL era, HMXBs were mostly BeXBs systems. For instance, in the catalogue of 130 HMXBs by Liu et al. (2000), there were 54 BeXBs (42\% of the total number of HMXBs) and 7 $\operatorname{sgXBs}(5 \%)$. Then, the situation changed with the first HMXBs identified by INTEGRAL: in the catalogue of HMXBs of Liu et al. (2006), out of 114 HMXBs (+128 in Magellanic Clouds), there were $60 \%$ of BeXBs and $32 \%$ of sgXBs firmly identified. Therefore, the ratio of BeXBs/HMXBs increased by a small factor of 1.5 , while the one of $\operatorname{sgXBs} / \mathrm{HMXBs}$ increased by a much higher factor of 6 .

We will see later that the two highlights of INTEGRAL in the XRB domain are first the emergence of an obscured population of $\operatorname{sgXBs}$, and then the emergence of the SFXT class (12 candidates).

In the 3 following subsections we follow the study reported in the exhaustive and excellent paper of Bodaghee et al. (2007).

\subsection{The Galactic spatial distribution}

We first examine the impact of stellar evolution of massive binaries on the formation of binary systems, by looking at the spatial distribution of binary systems with known distances on a Galactic spiral 4-arm model (based on locations of star-forming regions -SFRs- and related complexes: OB stars, molecular clouds, HII regions, diffuse ionised gas; see Russeil 2003).

The LMXBs, hosting old companion stars which had the time to migrate off the Galactic plane $\left(|\mathrm{b}|>3-5^{\circ}\right)$, are found to be concentrated in or near the Galactic bulge where old globular clusters reside, peaking at the center and decreasing gradually with the galactocentric radius, suggesting an association with the Galactic bar.

On the contrary, HMXBs, hosting young companion stars, are encountered in recent stellar formation sites, in the outer disk and arms where young stars are formed, following the HII/CO distribution. Underabundant in central few kpc, their uneven distribution along the Galactic plane reflects the Galactic spiral structure. Indeed, they coincide with the active young massive SFRs, peaking at $l \sim \pm 30^{\circ}$ towards tangential directions of inner spiral arm tangents (Norma and Scutum/Sagittarius) and towards a molecular ring located at $\sim 3 \mathrm{kpc}$ from the Galactic center. 
The spread of latitude distributions from the Galactic plane is larger in LMXBs than in HMXBs due to the relative young HMXB companions. Such evolutionary signatures had been already noticed by Ginga (Koyama et al., 1990) and RXTE (Grimm et al., 2002) but on smaller samples. However we point out that the exposure map is heterogeneous. According to their angular distribution and transience, the population of yet unclassified sources is likely composed primarily of Galactic LMXBs/CVs. The distribution of miscellaneous sources is similar to HMXBs.

Since the propagation of density waves promotes stellar formation in spiral arms (Lin et al., 1969), the distribution of HMXBs is offset with directions of spiral arm tangents because it requires $\sim 10 \mathrm{Myr}$ before one of the stars in a binary system collapses into a NS/BH. The Galactic rotation induces changes in the apparent positions of the arms, causing a delay between star formation epoch and time of maximum number of HMXBs. Therefore the currently active star-forming sites should be about $\sim 40^{\circ}$ away from regions which were active $10 \mathrm{Myr}$ ago and produced the current HMXBs (Lutovinov et al. 2005; Dean et al. 2005; Bodaghee et al. 2007).

\subsection{Absorption}

IGRs column densities are higher (by a factor of $\sim 4$ ) than expected from the radio maps. Previously known sources had $\left\langle N_{\mathrm{H}}\right\rangle=1.2 \times 10^{22} \mathrm{~cm}^{-2}$, while IGRs exhibit $<N_{\mathrm{H}}>=4.8 \times 10^{22} \mathrm{~cm}^{-2}$. The Galactic IGRs are HMXBs with high $N_{\mathrm{H}}\left(\sim 10^{23} \mathrm{~cm}^{-2}\right)$. The question which arises is therefore: are the Galactic IGRs intrinsically absorbed due to the geometry of the absorbing material, or extrinsically due to their location along the dusty Galactic plane? While the line-of-sight absorption shows that there are potential clustering or asymmetries in the local distribution of matter, the highest value of Galactic $N_{\mathrm{H}}$ is $\sim 3 \times 10^{22} \mathrm{~cm}^{-2}$ (Dickey \& Lockman, 1990). It seems therefore that the high absorption is intrinsic to IGRs. ISGRI ( $>20 \mathrm{keV})$ is immune to absorption that prevented discovery of absorbed sources with earlier soft X-ray telescopes.

The most heavily-absorbed Galactic sources $\left(N_{\mathrm{H}}>10^{23} \mathrm{~cm}^{-2}\right)$ are found in the direction towards the Norma Arm region, the most active formation site of young supergiant stars (Bronfman et al., 1996) -precursors to the absorbed HMXBs- and the Galactic Bulge and Scutum/Sagittarius arms.

\subsection{Modulations}

Strong magnetic fields in NS XRBs produce non-spherically symmetric emission patterns: due to misaligned magnetic and rotation axes, pulsations are seen in the X-ray light curve. Most IGRs have spin periods $P_{\text {spin }}=100-1000$ s, i.e. 10 times longer than $<P_{\text {spin }}>$ of pre-IGRs.

IGRs exhibiting extreme modulations are:

- IGR J00291+5934, with $P_{\text {spin }}=1.7 \mathrm{~ms}$, is the fastest accretion-powered pulsar ever observed (Galloway et al., 2005),

- IGR J16358 - 4726, with $P_{\text {spin }}=6000$ s, is the slowest NS rotator (Lutovinov et al. 2005; Patel et al. 2007).

Why do HMXB IGRs have longer pulse periods? $\operatorname{sgXBs}$ are wind-fed systems with strong magnetic fields that tend to have the longest pulse periods (e.g. Corbet 1984). INTEGRAL and 
$X M M-N e w t o n$ feature long orbital periods around Earth. The distribution of IGR $P_{\text {orb }}$ exhibit a bimodal shape similar to the one known before INTEGRAL, with 2 populations: LMXBs (and CVs, SNRs...) exhibit shorter $P_{\text {orb }}$, and HMXBs longer $P_{\text {orb }}$. The Corbet $P_{\text {spin }}-P_{\text {orb }}$ diagramme shows that the majority of IGRs is located among other known wind-fed $\mathrm{sgXBs}$. BeXBs have in general longer $P_{\text {orb }}$ than sgXBs (this was already known, see e.g. Stella et al. 1986). INTEGRAL is therefore not just finding new HMXBs pulsars, but predominantly long-period sgXBs.

\subsection{Modulation vs Absorption}

The accretion affects the $P_{\text {spin }}$ of a NS, depending on the velocity at the corotation radius $\left(V_{c}\right)$ where the magnetic field regulates the motion of matter:

- if $v_{\mathrm{c}}>v_{\text {Kepler: }}$ the material is spun away, taking angular momentum, and the NS slows down due to "propellor mechanism" (Illarionov \& Sunyaev, 1975).

- if $v_{\mathrm{c}}<v_{\text {Kepler: }}$ the material is accreted onto the NS, which either spins up or down depending on the direction of the angular momentum with respect to the NS spin (Waters \& van Kerkwijk, 1989).

Since the spherically-symmetric accretion on a NS is driven by the wind of the companion star, the HMXB pulsar spin rate is regulated by the stellar wind angular momentum of the companion star.

The wind density of a supergiant star has the form $\rho(r) \propto r^{-2}$. For Be stars, because stellar winds have dense and slow equatorial outflows and thin fast polar winds (Lamers \& Waters, 1987), the wind density drops faster: $\rho(r) \propto r^{-3}$ (Waters et al., 1988). In addition, there are stronger density and velocity gradients inside the capture radius of the NS, in both radial and azimutal directions. Wind-fed accretion is therefore more efficient at delivering angular momentum to the NS in BeXBs than it is in sgXBs (Waters \& van Kerkwijk, 1989).

Given the density structures, and assuming a steady accretion rate with angular momentum of same direction as the NS spin, it reaches an equilibrium value $P_{\text {eq }}$ propto $\rho^{-3 / 7}$. However, the current $P_{\text {spin }}$ of NS in sgXBs are much longer than predicted and are closer to $P_{\text {eqs }}$ of the stellar winds while the star was still on the main sequence (Waters \& van Kerkwijk, 1989). On the other hand, the equilibrium $P_{\text {spin }}$ in Be systems is constantly adjusting to the changing conditions in the winds. As with sg systems, pulsars in Be systems are not currently spinning at $P_{\text {eq }}$ but reflect the values of an earlier evolutionary stage (King, 1991). The transport of positive angular momentum through the wind is so inefficient that it can not spin up the pulsar to its expected equilibrium $P_{e q s}$. However the pulsar can be spun down by the "propellor mechanism".

\subsubsection{A new tool}

Galactic HMXB IGRs segregate into distinct regions of $P_{\text {spin }}-N_{\mathrm{H}}$ diagramme: $\operatorname{sgXBs}$ have higher average $N_{\mathrm{H}}$ and longer average $P_{\text {spin }}$ compared to BeXBs. There are only $2 \mathrm{sgXBs}$ with $P_{\text {spin }}$ $<50$ s: Cen X-3 a Roche-lobe overflow system and OAO 1657 - 415, which seem to be intermediate systems, since they show transitions from wind-fed to disk-fed system. 
In view of all what has been described until now, looking at the location of IGRs in a $P_{\mathrm{spin}}-N_{\mathrm{H}}$ diagramme constitutes a new tool to distinguish between sg and BeXBs when only $N_{\mathrm{fH}}$ and either $P_{\text {spin }}$ or $P_{\text {orb }}$ are known.

- IGR J19140+0951 ( $\left.P_{\text {orb }} \sim 13 d, N_{\mathrm{H}} \sim 10^{23} \mathrm{~cm}^{-2}\right)$ : positioned among $\mathrm{sgXBs}$, the sgOB spectral type has been confirmed by IR observations (see e.g. Chaty et al. 2008; and see also Prat et al. 2008 for the dependence of $N_{\mathrm{H}}$ along the phase);

- IGR J16358 - 4726: exhibiting an unusually long $P_{\text {spin }}$ suggesting a magnetar nature (Patel et al., 2007), and located in the sgXB part, the spectral type has been confirmed by Chaty et al. (2008).

\section{Now that INTEGRAL is orbiting... let the show go on!}

The INTEGRAL observatory has performed a detailed survey of the Galactic plane. The ISGRI detector on the IBIS imager has discovered many new high energy celestial objects, most of which have been reported in Bird et al. (2007) $)^{2}$. The most important result of INTEGRAL to date is the discovery of many new high energy sources - concentrated in the Galactic plane, and towards the Norma arm, a region of our Galaxy full of star forming regions, - exhibiting common characteristics which previously had rarely been seen (see e.g. Chaty \& Filliatre 2005). Many of them are high mass X-ray binaries (HMXBs) hosting a NS orbiting around an OB companion, in most cases a supergiant star. They divide into two classes: some of the new sources are very obscured, exhibiting a huge intrinsic and local extinction, -the most extreme example is the highly absorbed source IGR J16318-4848 (Filliatre \& Chaty, 2004)-, and the others are HMXBs hosting a supergiant star and exhibiting fast and transient outbursts - an unusual characteristic among HMXBs. These are therefore called Supergiant Fast X-ray Transients (SFXTs, Negueruela et al. 2006), with IGR J17544-2619 being their archetype (Pellizza et al., 2006).

Nearly all the INTEGRAL HMXBs for which both spin and orbital periods have been measured are located in the upper part of the Corbet diagramme (Corbet, 1986). They are wind accretors, typical of supergiant HMXBs, and X-ray pulsars exhibiting longer pulsation periods and higher absorption (by a factor $\sim 4$ ) as compared to the average of previously known HMXBs (Bodaghee et al., 2007). This extra absorption might be due to the presence of a cocoon of dust/cold gas enshrouding the whole binary system in the case of the obscured sources. The intrinsic properties of the supergiant companion star could therefore explain some properties of these sources. However, differences exist between obscured sources and SFXTs, which might be explained by the geometry of the binary systems, and/or the extension of the wind/cocoon enshrouding either the companion star or the whole system. Indeed, obscured sources are naturally explained by a compact object orbiting inside a cocoon of dust and/or cold gas, while the fast X-ray behaviour of SFXTs needs a clumpy stellar wind environment, to account for fast and transient accretion phenomena (see Figure 2, left and right panels respectively, and Chaty \& Rahoui 2006).

How to reveal the nature of these newly discovered IGRs? High-energy observations are not sufficient to reveal their nature, since the INTEGRAL/ISGRI localisation $(\sim z)$ is not accurate

\footnotetext{
${ }^{2}$ See an updated list at http://isdc.unige.ch/ rodrigue/html/igrsources.html
} 
enough to unambiguously pinpoint and identify the counterpart at other wavelengths. Once X-ray satellites such as XMM-Newton, Chandra, or Swift provide an arcsecond position, the hunt for the optical counterpart of the source is open. However, the high level of absorption due to interstellar dust and gas towards the Galactic plane/centre makes the near-infrared (NIR) domain more efficient for identifying these sources.

On the other hand, multi-wavelength observations allow us to study various components of the system, emitting in various wavelength domains, depending on the nature of the sources. There is a complementarity of telescopes from space to ground, starting with the discovery of the high energy source by $\mathrm{X} / \gamma$-ray satellites such as INTEGRAL, and then its localization in X-rays by XMMNewton, Swift, or even Chandra, bringing a sub-arcsecond localisation. After this localization, the counterpart can be further looked for in radio (VLA), optical and/or infrared wavelengths (for instance using ESO facilities).

In this section we now report on multi-wavelength observations of a sample of IGRs, belonging to both classes described above, and we then give general results on IGRs, before discussing them and concluding.

\subsection{Multi-wavelength observations of INTEGRAL Sources}

To better characterize this population, Chaty et al. (2008), Rahoui et al. (2008) and Tomsick et al. (2007) studied a sample of 21 IGRs belonging to both classes described above. IGRs of this sample are X-ray pulsars, with high $P_{\text {spin }}$ from 139 to 5880 s and $P_{\text {orb }}$ ranging from 4 to 14 days. They are therefore HMXBs wind accreting supergiants, according to the Corbet diagramme. The multiwavelength observations were performed from 2004 to 2008 at the European Southern Observatory (ESO), using Target of Opportunity (ToO) and Visitor modes, in 3 domains: optical $(400-800 \mathrm{~nm})$ with EMMI, NIR $(1-2.5 \mu \mathrm{m})$ with SOFI, both instruments at the focus of the $3.5 \mathrm{~m}$ New Technology Telescope (NTT) at La Silla, and mid-infrared (MIR, 5-20 $\mu \mathrm{m}$ ) with the VISIR instrument on Melipal, the 8m Unit Telescope 3 (UT3) of the Very Large Telescope (VLT) at Paranal (Chile). They also used data from the GLIMPSE survey of Spitzer. With these observations they performed accurate astrometry, identification, photometry and spectroscopy on this sample of IGRs, aiming at identifying their counterparts and the nature of the companion star, deriving their distance, and finally characterising the presence and temperature of their circumstellar medium, by fitting their spectral energy distribution (SED).

Some results are reported in Table 1. Before describing some of these multi-wavelength results in detail, we first mention the main results of this study. 15 of these IGR sources were identified as HMXBs, and among them 12 HMXBs containing massive and luminous early-type companion stars. By combining MIR photometry, and fitting their optical-MIR SEDs, Rahoui et al. (2008) showed that (i) most of these sources exhibit an intrinsic absorption and (ii) three of them exhibit a MIR excess, which they suggest to be due to the presence of a cocoon of dust and/or cold gas enshrouding the whole binary system, with a temperature of $T_{d} \sim 1000 \mathrm{~K}$, extending on a radius of $R_{d} \sim 10 R_{\star}$ (see also Chaty \& Rahoui 2006). 


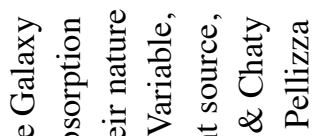

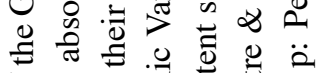
पे चै है

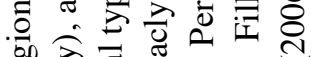
东 可 导

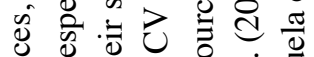
可过 ठ

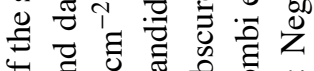

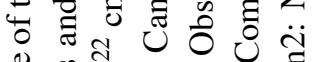
छี ङ

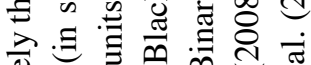

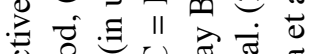

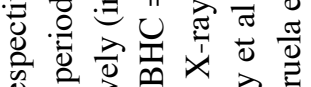

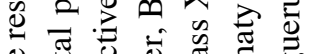

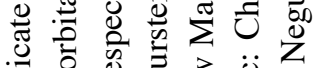
光

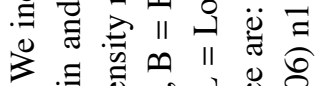
क्ष ठำ :

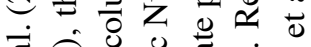

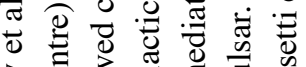

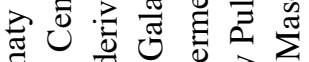
U. $\Xi$ 可 ฮ क्ष

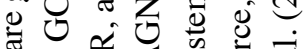

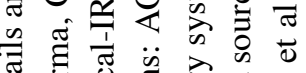

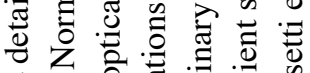

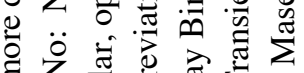

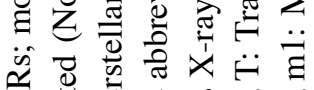

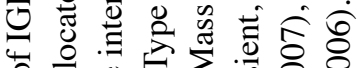
फ

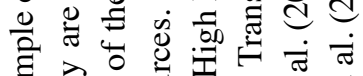

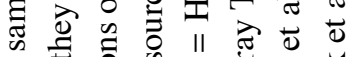
至

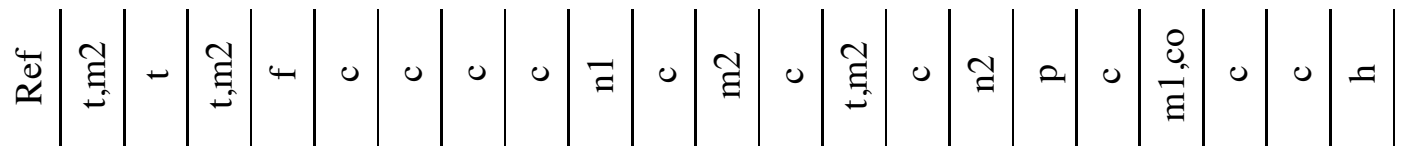

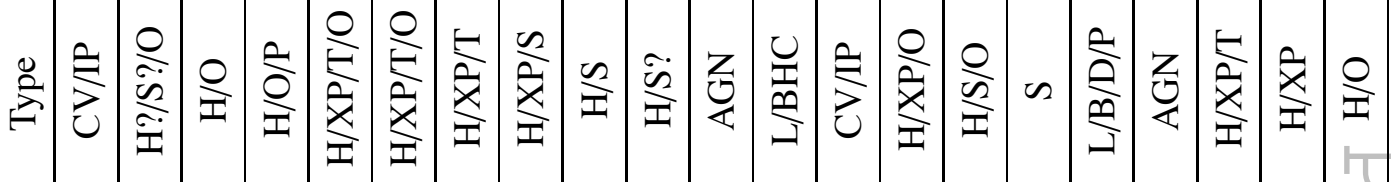

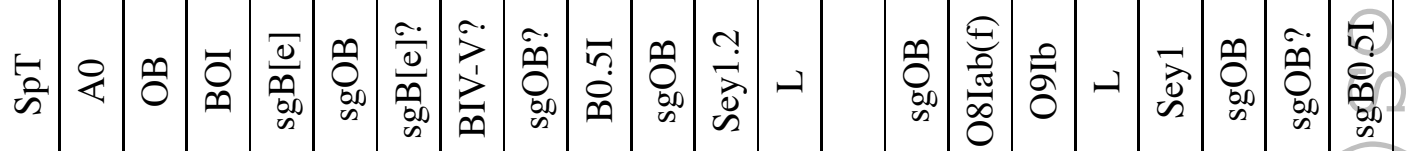

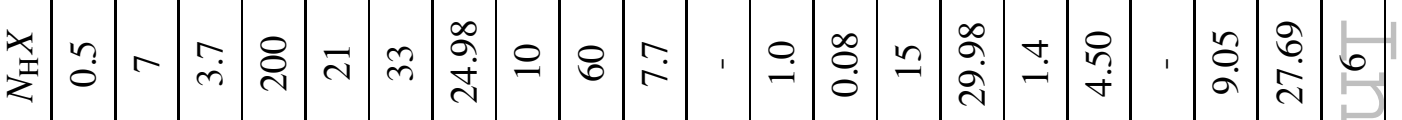

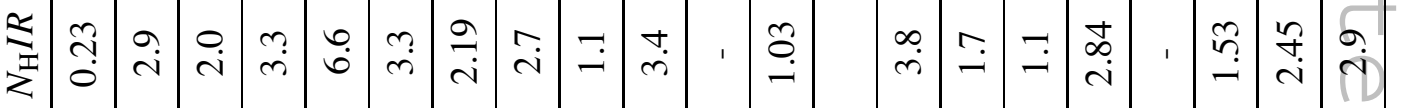

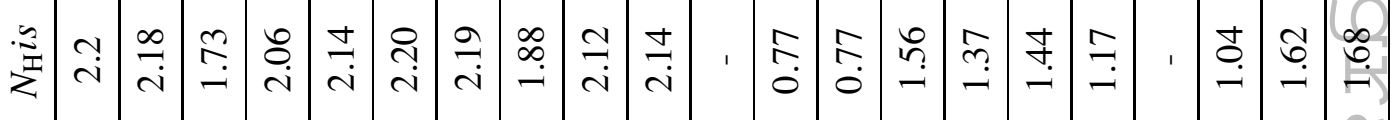

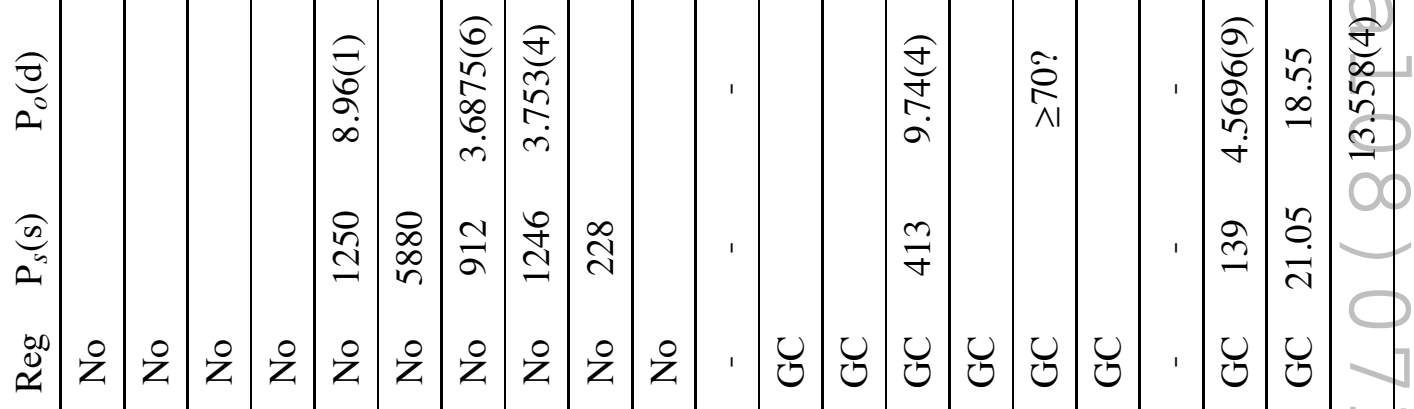

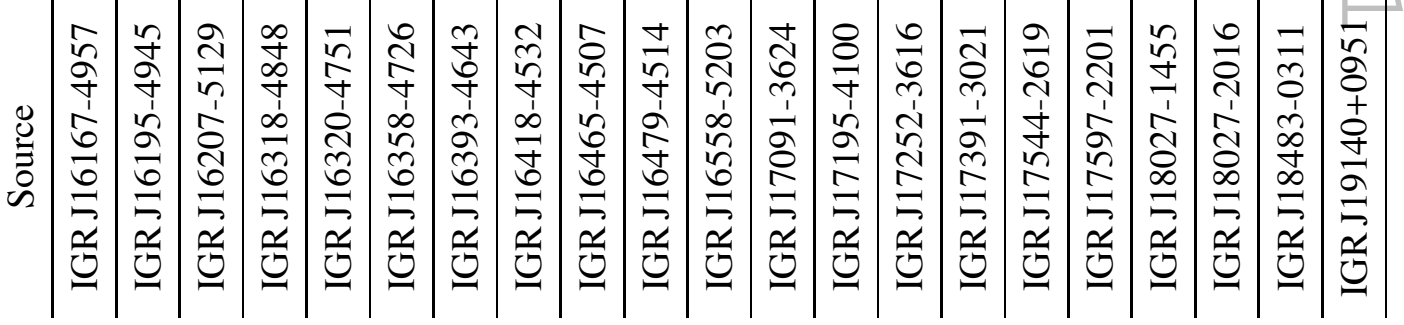

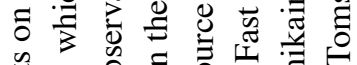

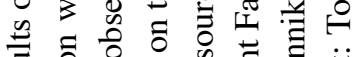
额 ․ㅡㅂ $\ddot{7}$ चु

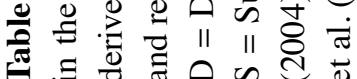




\subsection{Supergiant Fast X-ray Transients}

\subsubsection{General characteristics}

Supergiant Fast X-ray transients (also called "SFXTs") are high-mass X-ray binary systems hosting NS orbiting around sgOB companions. They constitute a new class of sources identified among the recently discovered IGRs, exhibiting the following common characteristics: rapid outbursts lasting only for hours, faint quiescent emission, high energy spectra requiring a BH or NS accretor, and supergiant OB companion stars. The flares have the following characteristics: their rise last for tens of minutes, the total duration is of $\sim 1$ hour, their frequency of $\sim 7$ days, and their luminosity $L_{x} \sim 10^{36} \mathrm{erg} \mathrm{s}^{-1}$ at the outburst peak. These sources are therefore characterized by general properties which are different from "classical" HMXBs.

\subsubsection{IGR J17544-2619: Archetype of the SFXTs}

IGR J17544-2619, a bright recurrent transient X-ray source discovered by INTEGRAL on 17 September 2003 (Sunyaev et al., 2003), seems to be their archetype. Observations with XMMNewton have shown that it exhibits a very hard X-ray spectrum, and a relatively low intrinsic absorption $\left(N_{\mathrm{H}} \sim 2 \times 10^{22} \mathrm{~cm}^{-2}\right.$, González-Riestra et al. 2004). Its bursts last for hours, and inbetween bursts it exhibits long quiescent periods, which can reach more than 70 days. The $\mathrm{X}$-ray behaviour is complex on long, mean and short-term timescales: rapid flares are detected by INTEGRAL on all these timescales, on pointed and 200s binned lightcurve (Zurita Heras \& Chaty, 2008b). The compact object is probably a NS (in't Zand, 2005). Pellizza et al. (2006) managed to get optical/NIR ToO observations only one day after the discovery of this source. They identified a likely counterpart inside the XMM-Newton error circle, confirmed by an accurate localization from Chandra. Spectroscopy showed that the companion star was a blue supergiant of spectral type O9Ib, with a mass of $25-28 M_{\odot}$, a temperature of $T \sim 31000 \mathrm{~K}$, and a stellar wind velocity of $265 \pm 20 \mathrm{~km} \mathrm{~s}^{-1}$ (which is faint for O stars): the system is therefore an HMXB (Pellizza et al., 2006). Rahoui et al. (2008) combined optical, NIR and MIR observations and showed that they could accurately fit the observations with a model of an O9Ib star, with a temperature $T_{\star} \sim 31000 \mathrm{~K}$ and a radius $R_{\star}=21.9 R_{\odot}$. They derived an absorption $\mathrm{A}_{v}=6.1$ magnitudes and a distance $\mathrm{D}=3.6 \mathrm{kpc}$. Therefore the source does not exhibit any MIR excess, it is well fitted by a unique stellar component (see Figure 1, right panel, Rahoui et al. 2008).

In summary, IGR J17544-2619 is an HMXB at a distance of $\sim 3.6 \mathrm{kpc}$, constituted of an O9Ib supergiant, with a mild stellar wind and a compact object which is probably a NS, without any MIR excess.

\subsubsection{Classification of SFXTs}

We can divide the SFXTs in two groups, according to the duration and frequency of their outbursts, and their $\frac{L_{\max }}{L_{\min }}$ ratio. The classical SFXTs exhibit a very low quiescence $L_{X}$, and a high variability, while intermediate SFXTs exhibit a higher $\left\langle L_{X}\right\rangle$, a lower $\frac{L_{\max }}{L_{\min }}$ and a smaller variability, with longer flares. SFXTs might appear like persistent $\operatorname{sgXBs}$ with $<L_{X}>$ below the canonical value of $\sim 10^{36} \mathrm{erg} \mathrm{s}^{-1}$, and flares superimposed. But there might be some observational biases, therefore the distinction between SFXTs and $\mathrm{sgXBs}$ is not well defined yet. While the typical hard $\mathrm{X}$-ray variability factor (the ratio between the deep quiescence to outburst flux) is less than 20 in 

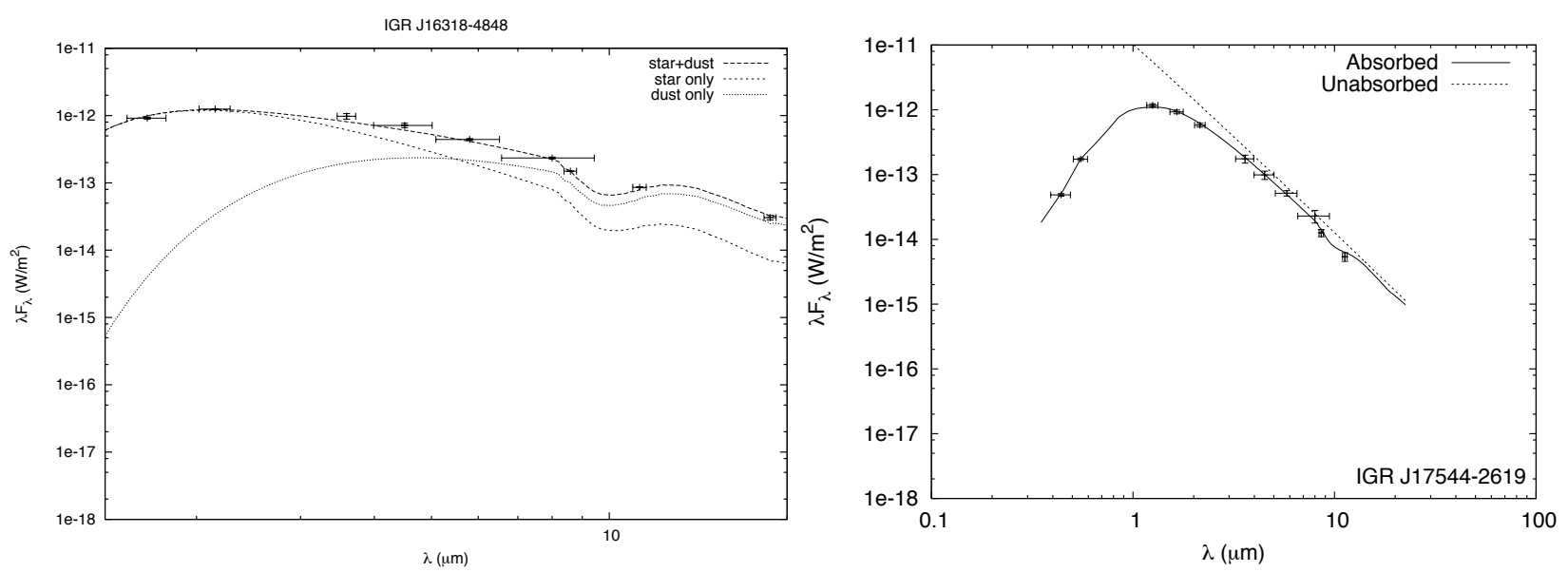

Figure 1: Optical to MIR SEDs of IGR J16318-4848 (left) and IGR J17544-2619 (right), including data from ESO/NTT, VISIR on VLT/UT3 and Spitzer (Rahoui et al., 2008). IGR J16318-4848 exhibits a MIR excess, interpreted by Rahoui et al. (2008) as the signature of a strong stellar outflow coming from the $\mathrm{sgB}[\mathrm{e}]$ companion star (Filliatre \& Chaty, 2004). On the other hand, IGR J17544-2619 is well fitted with only a stellar component corresponding to the O9Ib companion star spectral type (Pellizza et al., 2006).

classical/absorbed systems, it is higher than 100 in SFXTs (some sources can exhibit flares in a few minutes, like for instance XTEJ1739-302 \& IGR J17544-2619). The intermediate SFXTs exhibit smaller variability factors.

SFXT behaviour: clumpy wind accretion?

Such sharp rises exhibited by SFXTs are incompatible with the orbital motion of a compact object through a smooth medium (Negueruela et al. 2006, Smith et al. 2006, Sguera et al. 2005). Instead, flares must be created by the interaction of the accreting compact object with the dense clumpy stellar wind (representing a large fraction of stellar $\frac{d M}{d t}$ ). In this case, the flare frequency depends on the system geometry, and the quiescent emission is due to accretion onto the compact object of diluted inter-clumps medium, explaining the very low quiescence level in classical SFXTs.

To explain the emission of sgXBs/SFXTs, Negueruela et al. (2008) and Walter \& Zurita Heras (2007) invoke the existence of two zones around the supergiant star, of high and low clump density respectively. This would naturally explain the smooth transition between sgXBs and SFXTs, and the existence of intermediate systems; the main difference between the classical $\operatorname{sgXBs}$ and the SFXTs being in this scenario the NS orbital radius:

- The obscured sgXBs (persistent and luminous systems) would have short and circular orbit inside the zone of high clump density $\left(R_{o r b} \sim 2 R_{\star}\right)$.

- The intermediate SFXT would have short orbits, circular or eccentric, and possible periodic outbursts.

- The classical SFXT would have larger and eccentric orbital radius.

$\underline{\text { Macro-clumping scenario }}$ 
Each SFXT outburst is due to the accretion of a single clump, assuming that the X-ray lightcurve is a direct tracer of the wind density distribution. The typical parameters in this scenario are:

- a compact object with large orbital radius: $10 R_{\star}$,

- a clump size of a few tenths of $R_{\star}$,

- a clump mass of $10^{22-23} \mathrm{~g}$ (for $N_{\mathrm{H}}=10^{22-23} \mathrm{~cm}^{-2}$ ),

- a mass loss rate of $10^{-(5-6)} M_{\odot} / y r$,

- a clump separation of order $R_{\star}$ at the orbital radius,

- a volume filling factor: $0.02->0.1$

The flare to quiescent count rate ratio is directly related to the $\frac{\text { clumps }}{\text { inter-clump }}$ density ratio, which ranges between:

- 15-50 for intermediate SFXTs, and

- $10^{2-4}$ for "classical" SFXTs.

A very high degree of porosity (macroclumping) is required to reproduce the observed outburst frequency in SFXTs, in good agreement with UV line profiles and line-driven instabilities at large radii (Oskinova et al. 2007; Runacres \& Owocki 2005; Walter \& Zurita Heras 2007). The number of clumps vs radius in a ring of width $2 r_{a c c}$ and height 2 racc is given in Oskinova et al. (2007), for a velocity law with beta $=0.8$ and porosity parameter $L_{0}=0.35$.

\section{Difference sgXB/SFXT}

A basic model of porous wind predicts a substantial change in the properties of the wind "seen by the NS" at a distance $r \sim 2 R_{\star}$ (vertical asymptote in Figure 1 of Negueruela et al. 2008), where we stop seeing persistent $\mathrm{X}$-ray sources. There are 2-regimes:

- either the NS sees a large number of clumps, because it is embedded in a quasi-continuous wind;

- or the number density of clumps is so small that the NS is effectively orbiting in an empty space.

sgXBs can only lie within the two vertical dot-dashed lines (Negueruela \& Schurch, 2007).

\section{Classes of wind accretors}

In Negueruela \& Schurch (2007) Figure 2 represents the stellar wind of high (coloured area) and low (blank area) clump density respectively. The coloured are represents the left part of the asymptote in Figure 1 of the same paper. The HMXB configurations are:

- SFXTs on the left (circular orbit, NS outside the high density zone); 
- SFXTs on the right (highly eccentric orbit, longer quiescence intervals), with the NS grazing the coloured area at periastron (as IGR J00370+6122);

- Intermediate systems if the NS is inside the narrow transition zone;

- $\operatorname{sgXBs:}$ the NS is always inside the coloured area.

The observed division between $\operatorname{sgXBs}$ (persistent sgXBs, SFXTs, regular outbursters) is therefore naturally explained by simple geometrical differences in the orbital configurations.

\section{IGR J18483-0311: an intermediate SFXT?}

X-ray properties of this system were pointing towards an SFXT (Sguera et al., 2007), however it exhibits an unusual behaviour: its outbursts last for a few days (to compare to hours for classical SFXTs), and the ratio $L_{\max } / L_{\min } \sim 10^{3}$ (its quiescence is therefore at a higher level than the ratio $\sim 10^{4}$ for classical SFXTs). Moreover, its orbital period $P_{o r b}=18.5 \mathrm{~d}$ is low compared to classical SFXTs (with large/eccentric orbits). Finally, its orbital period and spin period $\left(P_{\text {spin }}=21.05 \mathrm{~s}\right)$ located it inbetween Be and sgXBs in the Corbet Diagramme, therefore in a ambiguous position. Rahoui \& Chaty (2008) identified the companion star of this system as a B0.5Ia supergiant, unambiguously showing that this system is an SFXT. Furthermore, they suggest that this system could be the first firmly identified intermediate SFXT, characterized by short, eccentric orbit (e between 0.4 and 0.6 according to Rahoui \& Chaty 2008), and long outbursts... This "intermediate" SFXT nature would explain its unusual characteristics among "classical" SFXTs.

\subsection{Obscured HMXBs}

\subsubsection{IGR J16318-4848: An extreme case}

IGR J16318-4848 was the first source discovered by IBIS/ISGRI on INTEGRAL on 29 January 2003 (Courvoisier et al., 2003), with a 2 uncertainty. XMM-Newton observations revealed a comptonised spectrum exhibiting an unusually high level of absorption: $N_{\mathrm{H}} \sim 1.84 \times 10^{24} \mathrm{~cm}^{-2}$ (Matt \& Guainazzi, 2003). The accurate localisation by XMM-Newton allowed Filliatre \& Chaty (2004) to rapidly trigger ToO photometric and spectroscopic observations in optical/NIR, leading to the confirmation of the optical counterpart (Walter et al., 2003) and to the discovery of the NIR one (Filliatre \& Chaty, 2004). The extremely bright NIR source $(B>25.4+/-1 ; \mathrm{I}=16.05+/-0.54$, $\mathrm{J}=10.33 \pm 0.14 ; \mathrm{H}=8.33 \pm 0.10$ and $\mathrm{Ks}=7.20 \pm 0.05$ magnitudes) exhibits an unusually strong intrinsic absorption in the optical $\left(A_{v}=17.4\right.$ magnitudes $), 100$ times stronger than the interstellar absorption along the line of sight $\left(A_{v}=11.4\right.$ magnitudes $)$, but still 100 times lower than the absorption in X-rays. This led Filliatre \& Chaty (2004) to suggest that the material absorbing in $\mathrm{X}$-rays was concentrated around the compact object, while the material absorbing in optical/NIR was enshrouding the whole system. The NIR spectroscopy in the $0.95-2.5 \mu \mathrm{m}$ domain allowed to identify the nature of the companion star, by revealing an unusual spectrum, with many strong emission lines:

- $\mathrm{H}, \mathrm{HeI}$ (P-Cyg) lines, characteristic of dense/ionised wind at a velocity of $400 \mathrm{~km} / \mathrm{s}$,

- HeII lines: the signature of a highly excited region, 
- [FeII]: reminiscent of shock heated matter,

- FeII: emanating from media of densities $>10^{5}-10^{6} \mathrm{~cm}^{-3}$,

- NaI: coming from cold/dense regions.

All these lines are originating from a highly complex and stratified circumstellar environment of various densities and temperatures, suggesting the presence of an envelope and strong stellar outflow responsible for the absorption. Only luminous early-type stars such as supergiant $\mathrm{sgB}[\mathrm{e}]$ show such extreme environments, and Filliatre \& Chaty (2004) concluded that IGR J16318-4848 was such an unusual HMXB, hosting a $\mathrm{sgB}[\mathrm{e}]$ with characteristic luminosity of $10^{6} L_{\odot}$ and mass of $30 M_{\odot}$ (see also Chaty \& Filliatre 2005).

The question of this huge absorption was still pending, and only MIR observations would allow to solve this question, and understand its origin. By combining these optical and NIR data with new MIR observations, and fitting these observations with a model of a $\operatorname{sgB}[\mathrm{e}]$ companion star, Rahoui et al. (2008) showed that IGR J16318-4848 was exhibiting a MIR excess (see Figure 1 , left panel), that they interpreted as being due to the strong stellar outflow emanating from the $\operatorname{sgB}[\mathrm{e}]$ companion star. They found that the companion star had a temperature of $T_{\star}=22200 \mathrm{~K}$ and radius $R_{\star}=20.4 R_{\odot}=0.1$ a.u., consistent with a supergiant star, and an extra component of temperature $\mathrm{T}=1100 \mathrm{~K}$ and radius $\mathrm{R}=11.9 R_{\star}=1$ a.u., with $\mathrm{A}_{v}=17.6$ magnitudes. Recent MIR spectroscopic observations with VISIR at the VLT showed that the source was exhibiting strong emission lines of $\mathrm{H}, \mathrm{He}, \mathrm{Ne}, \mathrm{PAH}, \mathrm{Si}$, proving that the extra absorbing component was made of dust and cold gas.

By taking a typical orbital period of 10 days and a mass of the companion star of $20 \mathrm{Mb}$, we obtain an orbital separation of $50 R_{\odot}$, smaller than the extension of the extra component of dust/gas (= $240 R_{\odot}$ ), suggesting that this component enshrouds the whole binary system, as would do a cocoon of gas/dust (see Figure 2, left panel).

In summary, IGR J16318-4848 is an HMXB system, located at a distance between 1 and $6 \mathrm{kpc}$, hosting a compact object (probably a NS) and a $\operatorname{sgB}[\mathrm{e}]$ star (it is therefore the second HMXB with a sgB[e] star, after CI Cam; see Clark et al. 1999). The most striking facts are (i) the compact object seems to be surrounded by absorbing material and (ii) the whole binary system seems to be surrounded by a dense and absorbing circumstellar material envelope or cocoon, made of cold gas and/or dust. This source exhibits such extreme characteristics that it might not be fully representative of the other obscured sources.

\subsection{The Grand Unification: different geometries, different scenarios}

In view of the results described above, there seems to be a continuous trend, from classical and/or absorbed sgHMBs, to classical SFXTs. We outline in the following this trend.

- In "classical" sgXBs, the NS is orbiting at a few stellar radii only from the star. The absorbed (or obscured) sgXBs (like IGR J16318-4848) are classical sgXBs hosting NS constantly orbiting inside a cocoon made of dust and/or cold gas, probably created by the companion star itself. These systems therefore exhibit a persistent X-ray emission. The cocoon, with an extension of $\sim 10 R_{\star}=1$ a.u., is enshrouding the whole binary system. The NS has a small and circular orbit (see Figure 2, left panel). 
- In "Intermediate" SFXT systems (such as IGR J18483-0311), the NS orbits on a small and circular/excentric orbit, and it is only when the NS is close enough to the supergiant star that accretion takes place, and that $\mathrm{X}$-ray emission arises.

- In "classical" SFXTs (such as IGR J17544-2619), the NS orbits on a large and excentric orbit around the supergiant star, and exhibits some recurrent and short transient X-ray flares, while it comes close to the star, and accretes from clumps of matter coming from the wind of the supergiant. Because it is passing through more diluted medium, the $\frac{L \max }{L \min }$ ratio is higher for "classical" SFXTs than for "intermediate" SFXTs (see Figure 2, right panel).

Although this scenario seems to describe quite well the characteristics currently seen in sgXBs, we still need to identify the nature of many more sgXBs to confirm this scenario, and in particular the orbital period and the dependance of the column density with the phase of the binary system.
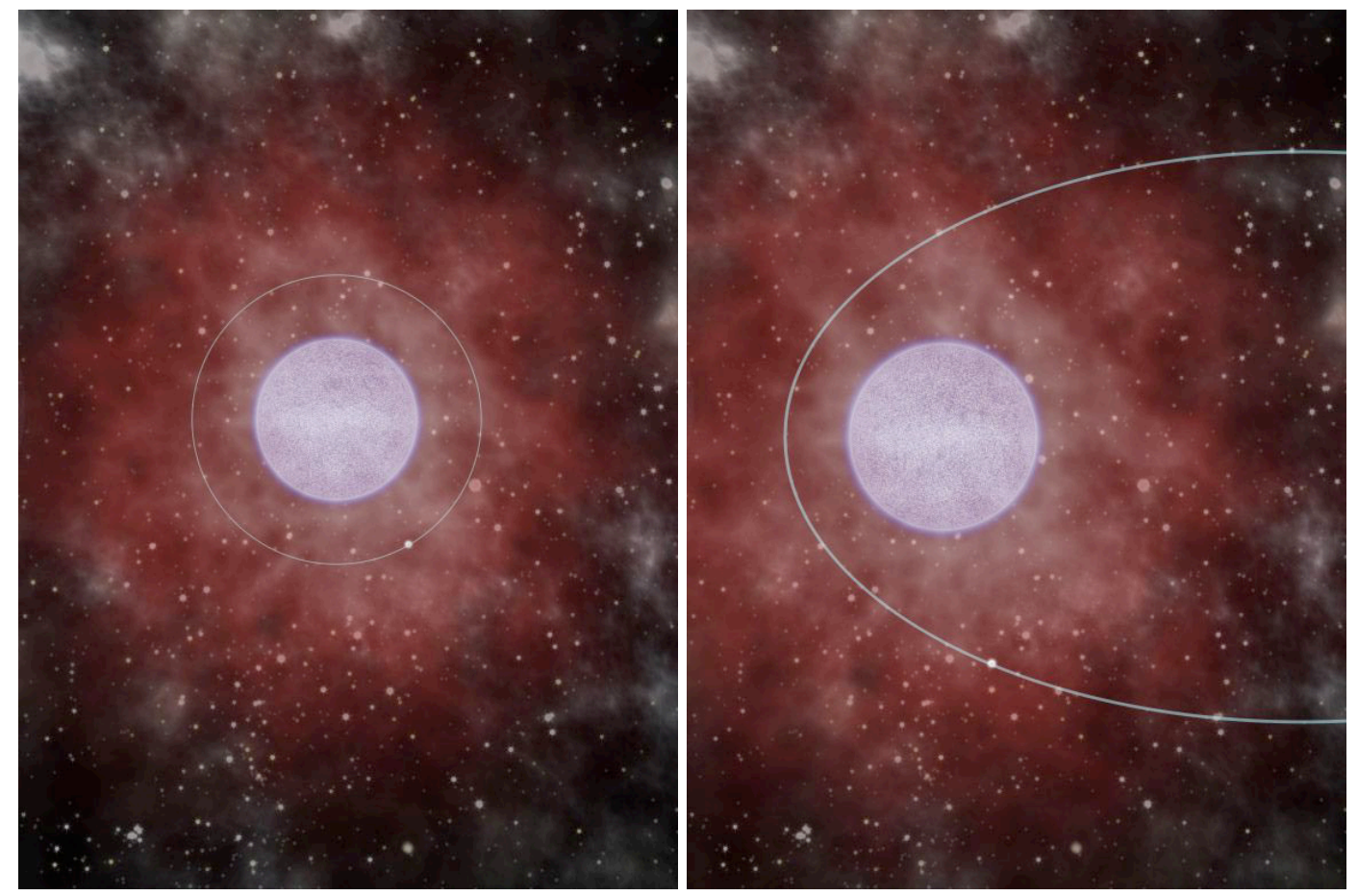

Figure 2: Scenarios illustrating two possible configurations of INTEGRAL sources: a NS orbiting a supergiant star on a circular orbit (left image); and on an eccentric orbit (right image), accreting from the clumpy stellar wind of the supergiant. The accretion of matter is persistent in the case of the obscured sources, as in the left image, where the compact object orbits inside the cocoon of dust enshrouding the whole system. On the other hand, the accretion is intermittent in the case of SFXTs, which might correspond to a compact object on an eccentric orbit, as in the right image. A 3D animation of these sources is available on the website: http://www.aim.univ-paris7.fr/CHATY/Research/hidden.html

\subsubsection{Obscured Be X-ray binary systems?}

Now that we have described the obscured sgXBs, we point out that we might have found the most highly absorbed and distant Be star in a HMXB system: AX J1749.1-2733. This system is an HMXB pulsar, with a $P_{\text {orb }}=185 \mathrm{~d}$ and a $P_{\text {spin }}=66 \mathrm{~s}$, and flares lasting for 12 days. Its characteristics 
are therefore typical of Be star in HMXB pulsars. Zurita Heras \& Chaty (2008a) have identified its optical counterpart: it is a Be star exhibiting a high level of absorption $\left(N_{\mathrm{H}}=2 \times 10^{23} \mathrm{~cm}^{-2}\right)$, located far away in our Galaxy (> 8.5 kpc), probably similar to the BeXB 2S 1845-024.

\subsubsection{Population synthesis models}

These sources revealed by INTEGRAL, namely the supergiant HMXBs, will allow to give constraints and finally to better understand the formation and evolution of binary systems, by comparing them to numerical study of LMXB/HMXB population synthesis models. Many parameters do influence the various evolutions of these systems: differences in size, orbital period, ages, accretion type, and stellar endpoints... Moreover, stellar and circumstellar properties also influence the evolution of high-energy binary systems, made of two massive components usually born in rich star forming regions. Finally, these new systems might represent a precursor stage of what is known as the "Common envelope phase" in the evolution of LMXB/HMXB systems.

These sources are also useful to look for massive stellar "progenitors", for instance giving birth to coalescence of compact objects, through NS/NS or NS/BH collisions. They would then become prime candidate for gravitational wave emitters, or even to short/hard $\gamma$-ray bursts.

\section{Conclusions and perspectives...}

The INTEGRAL satellite has tripled the total number of Galactic $\operatorname{sgXBs,~constituted~of~a~NS~}$ orbiting around a supergiant star. Most of these new sources exhibit a large $N_{\mathrm{H}}$ and long $P_{\text {spin }}$ $(\sim 1 \mathrm{ks})$ : they are slow and absorbed X-ray pulsars. The influence of the local absorbing matter on periodic modulations is different for sg or BeXBs: sgOB or BeXBs are segregated in different parts of $N_{\mathrm{H}^{-}} P_{\text {orb }}$ or $N_{\mathrm{H}^{-}} P_{\text {spin }}$.

INTEGRAL revealed 2 new types of sources. First, the SFXTs (Supergiant Fast X-ray Transients) exhibiting short and strong X-ray flares, with a peak flux of 1 Crab during 1-100s, every $\sim 100$ days. These short and intense flares can be explained by accretion through clumpy winds. Second, the obscured HMXBs are composed of supergiant stellar companions exhibiting a strong intrinsic absorption. They are X-ray pulsars with persistent emission, and long $P_{\text {spin }}$. The NS is deeply embedded in the dense stellar wind, which forms a dust cocoon enshrouding the whole binary system.

These results show the existence in our Galaxy of a dominant population of a previously rare class of high-energy binary systems: supergiant HMXBs, some exhibiting a high intrinsic absorption (Chaty et al. 2008; Rahoui et al. 2008). A careful study of this population, recently revealed by INTEGRAL, will provide a better understanding of the formation and evolution of short-living HMXBs. Furthermore, stellar population models will henceforth have to take these objects into account, to assess a realistic number of high-energy binary systems in our Galaxy. Our final word is that only a multiwavelength study reveal the nature of these obscured high-energy sources.

The recently successfully launched GLAST satellite (Gamma-ray Large Area Space Telescope, operating in the $10 \mathrm{keV}-300 \mathrm{GeV}$ energy range) will certainly discover such new and unexpected objects. Indeed, although these $\mathrm{sgXB}$ sources do not seem to be GeV Emitters, there are peculiar members of this family which emit at these energy ranges, for instance LSI +61 303 
(a "disguised" pulsar, Dubus 2006), LS 5039 (Paredes et al. 2000; Aharonian et al. 2005), and Cygnus X-1 (Albert et al., 2007). This high energy satellite has a huge potential: 200 individual sources have been detected by EGRET... we expect that thousands will be detected by GLAST!

\section{Acknowledgments}

SC would like to thank the organisers of this successful workshop for their invitation to give this contributed talk on the newly discovered INTEGRAL sources in the nice city of Copenhaguen!

\section{References}

Aharonian, F., Akhperjanian, A. G., Aye, K.-M., et al. 2005, Science, 309, 746

Albert, J., Aliu, E., Anderhub, H., et al. 2007, ApJL, 665, L51

Bird, A. J., Malizia, A., Bazzano, A., et al. 2007, ApJSS, 170, 175

Bodaghee, A., Courvoisier, T. J.-L., Rodriguez, J., et al. 2007, A\&A, 467, 585

Bronfman, L., Nyman, L.-A., \& May, J. 1996, A\&ASS, 115, 81

Chaty, S. \& Filliatre, P. 2005, Astrophysics \& Space Sciences, 297, 235

Chaty, S. \& Rahoui, F. 2006, in The Obscured Universe, Procs. of 6th INTEGRAL workshop, Moscow, Russia, July 2-8, 2006, to be published by ESA's Publications Division in December 2006 as Special Publication SP-622, in press (astro-ph/0609474)

Chaty, S., Rahoui, F., Foellmi, C., et al. 2008, A\&A, 484, 783

Clark, J. S., Steele, I. A., Fender, R. P., \& Coe, M. J. 1999, A\&A, 348, 888

Coe, M. J. 2000, in ASP Conf. Ser. 214: IAU Colloq. 175: The Be Phenomenon in Early-Type Stars, ed. M. A. Smith, H. F. Henrichs, \& J. Fabregat, 656-+

Combi, J. A., Ribó, M., Martí, J., \& Chaty, S. 2006, A\&A, 458, 761

Corbet, R. H. D. 1984, A\&A, 141, 91

Corbet, R. H. D. 1986, MNRAS, 220, 1047

Courvoisier, T. J.-L., Walter, R., Rodriguez, J., Bouchet, L., \& Lutovinov, A. A. 2003, IAU Circ., 8063,3

Dean, A. J., Bazzano, A., Hill, A. B., et al. 2005, A\&A, 443, 485

Dickey, J. M. \& Lockman, F. J. 1990, ARA\&A, 28, 215

Dubus, G. 2006, A\&A, 456, 801

Filliatre, P. \& Chaty, S. 2004, ApJ, 616, 469 
Galloway, D. K., Markwardt, C. B., Morgan, E. H., Chakrabarty, D., \& Strohmayer, T. E. 2005, ApJL, 622, L45

Giacconi, R., Gursky, H., Paolini, F. R., \& Rossi, B. B. 1962, Physical Review Letters, 9, 439

González-Riestra, R., Oosterbroek, T., Kuulkers, E., Orr, A., \& Parmar, A. N. 2004, A\&A, 420, 589

Grimm, H.-J., Gilfanov, M., \& Sunyaev, R. 2002, A\&A, 391, 923

Hannikainen, D. C., Rawlings, M. G., Muhli, P., et al. 2007, MNRAS, 380, 665

Illarionov, A. F. \& Sunyaev, R. A. 1975, A\&A, 39, 185

in't Zand, J. J. M. 2005, A\&A, 441, L1

Kaper, L., van der Meer, A., \& Tijani, A. H. 2004, in Revista Mexicana de Astronomia y Astrofisica Conference Series, ed. C. Allen \& C. Scarfe, 128-131

King, A. R. 1991, MNRAS, 250, 3P

Koyama, K., Kawada, M., Kunieda, H., Tawara, Y., \& Takeuchi, Y. 1990, Nature, 343, 148

Lamers, H. J. G. L. M. \& Waters, L. B. F. M. 1987, A\&A, 182, 80

Lebrun, F., Terrier, R., Bazzano, A., et al. 2004, Nature, 428, 293

Lin, C. C., Yuan, C., \& Shu, F. H. 1969, ApJ, 155, 721

Liu, Q. Z., van Paradijs, J., \& van den Heuvel, E. P. J. 2000, Astron. Astrophys. Suppl. Ser., 147, 25

Liu, Q. Z., van Paradijs, J., \& van den Heuvel, E. P. J. 2006, A\&A, 455, 1165

Liu, Q. Z., van Paradijs, J., \& van den Heuvel, E. P. J. 2007, A\&A, 469, 807

Lutovinov, A., Revnivtsev, M., Gilfanov, M., et al. 2005, A\&A, 444, 821

Masetti, N., Morelli, L., Palazzi, E., et al. 2006, A\&A, 459, 21

Masetti, N., Palazzi, E., Bassani, L., Malizia, A., \& Stephen, J. B. 2004, A\&A, 426, L41

Matt, G. \& Guainazzi, M. 2003, MNRAS, 341, L13

Negueruela, I. 2004, in Revista Mexicana de Astronomia y Astrofisica Conference Series, ed. G. Tovmassian \& E. Sion, 55-56

Negueruela, I. \& Schurch, M. P. E. 2007, A\&A, 461, 631

Negueruela, I., Smith, D. M., \& Chaty, S. 2005, The Astronomer's Telegram, 429

Negueruela, I., Smith, D. M., Reig, P., Chaty, S., \& Torrejón, J. M. 2006, in ESA Special Publication, Vol. 604, ESA Special Publication, ed. A. Wilson, 165-170 
Negueruela, I., Torrejon, J. M., Reig, P., Ribo, M., \& Smith, D. M. 2008, ArXiv e-prints, 0801.3863

Oskinova, L. M., Hamann, W.-R., \& Feldmeier, A. 2007, A\&A, 476, 1331

Paredes, J. M., Martí, J., Ribó, M., \& Massi, M. 2000, Science, 288, 2340

Patel, S. K., Zurita, J., Del Santo, M., et al. 2007, ApJ, 657, 994

Pellizza, L. J., Chaty, S., \& Negueruela, I. 2006, A\&A, 455, 653

Prat, L., Rodriguez, J., Hannikainen, D. C., \& Shaw, S. E. 2008, MNRAS, 389, 301

Rahoui, F. \& Chaty, S. 2008, ArXiv e-prints

Rahoui, F., Chaty, S., Lagage, P.-O., \& Pantin, E. 2008, A\&A, 484, 801

Runacres, M. C. \& Owocki, S. P. 2005, A\&A, 429, 323

Russeil, D. 2003, A\&A, 397, 133

Sguera, V., Barlow, E. J., Bird, A. J., et al. 2005, A\&A, 444, 221

Sguera, V., Hill, A. B., Bird, A. J., et al. 2007, A\&A, 467, 249

Smith, D. M., Heindl, W. A., Markwardt, C. B., et al. 2006, ApJ, 638, 974

Stella, L., White, N. E., \& Rosner, R. 1986, ApJ, 308, 669

Sunyaev, R. A., Grebenev, S. A., Lutovinov, A. A., et al. 2003, The Astronomer's Telegram, 190

Tomsick, J. A., Chaty, S., Rodriguez, J., et al. 2006, ApJ, 647, 1309

Tomsick, J. A., Chaty, S., Rodriguez, J., \& Walter, R. 2007, The Astronomer's Telegram, 1018

Walter, R., Rodriguez, J., Foschini, L., et al. 2003, A\&A, 411, L427

Walter, R. \& Zurita Heras, J. 2007, A\&A, 476, 335

Waters, L. B. F. M., van den Heuvel, E. P. J., Taylor, A. R., Habets, G. M. H. J., \& Persi, P. 1988, A\&A, 198, 200

Waters, L. B. F. M. \& van Kerkwijk, M. H. 1989, A\&A, 223, 196

Zurita Heras, J. A. \& Chaty, S. 2008a, A\&A

Zurita Heras, J. A. \& Chaty, S. 2008b, A\&A 